\title{
Etat actuel de la sécondarisation de la forêt en périphérie nord de la Réserve de biosphère du Dja (Sud-est Cameroun) : influences des facteurs anthropiques passés et des éléphants
}

\author{
Guy Merlin NGUENANG ${ }^{1 *}$, Bernard Aloys NKONGMENECK ${ }^{1}$, Jean-François GILLET ${ }^{2}$, \\ Cédric VERMEULEN ${ }^{2}$, Jef DUPAIN ${ }^{3}$ et Jean-Louis DOUCET ${ }^{2}$ \\ ${ }^{1}$ Laboratoire de Systématique et d'Ecologie Végétales, Université de Yaoundé 1. B.P. 812 Yaoundé, Cameroun. \\ ${ }^{2}$ Laboratoire de Foresterie des Régions Tropicales et Subtropicales, Unité de Gestion des Ressources \\ Forestières et des Milieux Naturels, Gembloux Agro-Bio Tech, Université de Liège. Passage des Déportés 2, \\ B-5030 Gembloux, Belgium. \\ ${ }^{3}$ Center for Research and Conservation, Royal Zoological Society of Atwerp, Koningin Astriplein 26, 2018 \\ Antwerp, Belgium. \\ *Auteur correspondant, E-mail : gnguenang@hotmail.com, Tel. (237)75947756.
}

\section{RESUME}

Plusieurs études récentes révèlent une proportion importante de formations secondaires au sein des forêts tropicales africaines. La présente étude a pour but, de caractériser les différents faciès de végétation de la forêt du Dja (Sud-est Cameroun) en ressortant l'état et les causes de sa secondarisation. L'étude a été menée dans deux sites en périphérie nord de la Réserve de biosphère du Dja, l'un situé hors de la Réserve (Mimpala) et l'autre à l'intérieur (Dingué). Un total de $104 \mathrm{~km}$ de transects a été parcouru. La caractérisation de la végétation a été faite le long de transects en relevant les différents faciès de végétation traversés. Les marques visibles d'anciennes présences humaines et les réseaux de pistes d'éléphants rencontrés ont été comptabilisés. Les chiffres révèlent une prédominance des formations secondarisées aussi bien dans le site situé à l'extérieur de la Réserve, que dans celui se trouvant dans la Réserve: soit respectivement $52 \%$ et $58 \%$. Notre étude permet de montrer que l'état actuel de la secondarisation de la forêt du Dja est fortement lié à l'action anthropique traditionnelle dans un passé plus ou moins récent et à celle des éléphants.

(C) 2010 International Formulae Group. All rights reserved.

Mots clés : Forêts tropicales, forêts secondaires, impact humain, conservation.

\section{INTRODUCTION}

La forêt camerounaise du Dja fait partie de la grande région Guinéo-congolaise, d'après la classification établie par White (1983). Elle se situe dans le domaine Camerouno-congolais en deçà des limites Sud de la forêt semi-caducifoliée (Letouzey, 1985). Elle apparaît comme une forêt mixte de transition entre la forêt sempervirente et la forêt semi-caducifoliée (Sonké, 1996).

Il apparait de plus en plus évident que les forêts tropicales africaines ont subi l'effet de perturbations anthropiques importantes depuis un passé lointain jusqu'à nos jours. L'ampleur de l'utilisation humaine de la forêt tropicale africaine a été plus importante que ce que l'on admettait jadis (Clist, 1990). 
L'évidence des activités humaines allant jusqu'à 3000 ans BP (Before Present) a été établie au travers diverses études anthracologiques et archéologiques (Oslisly et al., 1994 ; Hart et al., 1996; White et Oates, 1999 ; Mbida et al., 2001 ). Les termes «forêt primaire et forêt vierge » qui font référence aux forêts n'ayant subies aucune perturbation humaine sont aujourd'hui largement contreversés, tout autant que la notion de climax (Willis et al., 2004 ; Genot, 2006).

Van Gemerden et al. (2003), ont montré qu'il est possible de déceler l'origine d'une formation forestière issue des perturbations anthropiques passées, au regard de la présence de certaines espèces ligneuses dominant la canopée.

Les travaux d'Oslisly (2006) confirment que la prédominance de Lophira alata, espèce héliophile longévive, est liée à la forte présence historique de l'homme dans la forêt du littoral camerounais.

D'un autre côté, Il a été démontré que les éléphants des forêts (Loxodonta africana cyclotis), bien que jouant un rôle dans la dissémination des graines de plusieurs espèces végétales (White, 1995), ont une action perturbatrice sur la forêt (Hemborg et al., 2006). Au cours de leurs déplacements et pour leur nutrition, ils cassent fréquemment des arbres et arbustes dont ils se nourrissent. Leur préférence pour les trouées et les jeunes formations secondaires contribuerait à maintenir l'état de secondarisation de la forêt (Vanleeuwe et Gautier, 1998; Paul et al., 2004).

Plusieurs études botaniques ont été menées dans la forêt du Dja (Lejoly, 1995 ; Sonké, 1996 ; Mbolo, 2004). Cependant, les données sur l'état actuel de la secondarisation de cette forêt restent fragmentaires.

Dans le cadre de cette étude, nous nous sommes intéressés à faire sur la base d'une caractérisation simple de la végétation, un état de la secondarisation de la forêt en périphérie nord de la Réserve de faune du Dja. Le rôle de l'homme dans un passé plus ou moins récent, des éléphants et d'autres facteurs à l'origine de cette secondarisation est ensuite discuté. Les implications en matière d'aménagement et de conservation sont finalement exposées.

\section{MATERIEL ET METHODE}

\section{Site d'étude}

L'étude a été menée dans deux sites en périphérie nord de la Réserve de faune du Dja. La zone d'étude s'étend entre $2^{\circ} 40^{\prime}$ et $3^{\circ} 23^{\prime}$ de latitude Nord et entre $12^{\circ} 25^{\prime}$ et $13^{\circ} 35^{\prime}$ de longitude Est (Figure 1).

Le climat de la région du Dja est de type équatorial humide, caractérisé par l'alternance de 4 saisons (Ben Yahmed et al., 2005). La température moyenne mensuelle s'élève à $23{ }^{\circ} \mathrm{C}$. Les précipitations sont abondantes et s'élèvent à $1600 \mathrm{~mm}$ en moyenne par an. Les sols sont de type ferrallitique jaune ou rouge, à l'horizon ferrugineux indurés et profonds. L'altitude varie entre 400 et $700 \mathrm{~m}$ (Ben Yahmed et al., 2005).

La Réserve de faune du Dja couvre une superficie approximative de 526000 hectares et compte parmi les plus vastes zones protégées de la forêt du bassin du Congo (UNEP, 2004). Elle abrite une grande proportion de faune sauvage incluant les espèces menacées comme les éléphants (Loxodonta africana), les gorilles (Gorilla g. gorilla) et les chimpanzés (Pan troglodytes) (Williamson et Usungo, 1998).

Le premier site d'étude (Mimpala) est situé dans une UFA (Unité Forestière d'Aménagement), cédée actuellement en concession par l'Etat camerounais à la société forestière FIPCAM qui l'exploite. Ce site fait l'objet d'une exploitation forestière industrielle depuis les années 1972.

Le second site (Dingué) se trouve dans la Réserve de faune du Dja et n'a jamais fait l'objet d'une exploitation forestière industrielle.

Les deux sites sont situés à une quinzaine de kilomètre à vol d'oiseau l'un de l'autre. Ils sont tous deux éloignés des lieux d'habitation actuels. 


\section{Caractérisation de la végétation}

Des transects parallèles de $6 \mathrm{~km}$ et équidistants de $600 \mathrm{~m}$ ont été ouverts dans le site de Mimpala. Dans le site de Dingué, nous avons, pour des raisons d'efficience et de rendement optimal des guides locaux, revu la longueur et l'équidistance des transects à $5 \mathrm{~km}$ et $500 \mathrm{~m}$ respectivement. $104 \mathrm{~km}$ de transects au total ont été ouverts dont $54 \mathrm{~km}$ dans le site de Mimpala et $50 \mathrm{~km}$ dans le site de Dingué. Les transects sont marqués tous les $25 \mathrm{~m}$ par un jalon indicateur.

La caractérisation des différents faciès de la végétation a été faite le long de transects. La méthode d'étude de la végétation utilisée est inspirée de White et Edwards (2000). On note tout au long des transects, les types de végétation traversés et leurs distances.

Huit types de végétation ont été définis sur une base visuelle: $F D M$, forêt dense mature peu ou pas perturbée caractérisée par son sous-bois ouvert, avec la strate arbustive dominée par les espèces des genres Rinorea et Drypetes ; FDSA, forêt dense secondaire âgée, avec sous bois moins ouvert; $F D S$, forêt dense secondaire avec une canopée discontinue et la présence de grandes taches de Marantacées: (Ataenidia conferta, Megaphrynium macrostachyum) plus prononcées que la forêt dense secondaire âgée; FSJ, forêt secondaire jeune avec un sous-bois touffu, la strate dominante atteint à peine $15 \mathrm{~m} ; R A P$, raphiale sur sol hydromorphe caractérisée par une forte densité de Raphia mobutterum, plusieurs espèces de Marantaceae et Zingiberaceae à l'instar de Halopegea azura, Marantochloa purpurea; RIP, forêt ripicole, forêt de zone de transition entre la forêt de terre ferme et les raphiales, périodiquement inondée; $C L$, clairières sur sols hydromorphes ou prairies marécageuses, se retrouvent dans les enclaves mal drainées de la forêt; elles sont dominées par les espèces herbacées telles que Kyllinga tenuifolia; PI, anciennes pistes forestières, avec une forte densité de Marantaceae et de Zingiberaceae.
Inventaire floristique, réseaux de pistes d'éléphants et marques d'anciennes présences humaines

Un inventaire floristique a été fait sur les placettes circulaires de $5 \mathrm{~m}$ de rayon au début de chaque transect et à tous les $250 \mathrm{~m}$. Le type de végétation est noté à chaque fois. 435 placettes correspondant à une surface totale de 3,41 ha, ont été inventoriées. Tous les arbres de $\mathrm{dhp} \geq$ à $10 \mathrm{~cm}$ (diamètre à hauteur de poitrine, soit à $1,30 \mathrm{~m} \mathrm{du}$ sol) ont été inventoriés et identifiés. La hauteur de la canopée, c'est-à-dire la hauteur de la strate dont la cime continue est la plus élevée, a également été estimée à vue. Nous n'avons pas tenu compte des émergent qui dominent quelque fois cette canopée. Afin de minimiser la variation de l'erreur de mesure, toutes les estimations ont été faites par le même observateur.

Tout au long des transects, les réseaux de pistes d'éléphants traversés ont été comptés. Nous avons délibérément considéré comme appartenant à un même réseau, toutes les pistes d'éléphants situées à moins de 100 $m$ les unes des autres. Nous n'avons cependant pas fait de distinction des différents sentiers d'éléphants en fonction de leur importance comme ont pu le faire Vanleeuwe et al. (1998).

Nous avons aussi relevé les marques d'anciennes présences humaines visibles de part et d'autres à partir des transects parcourus. Ces indices sont essentiellement des vestiges de grandes fosses d'antan utilisées comme piège et appelés "ngùla" chez les Badjoués (Vermeulen, 2000), les populations de grands palmiers (Elaeis guineensis) en pleine forêt atteignant 25 à 30 $\mathrm{m}$ de hauteur; de vieux individus restant d'anciennes parasoleraies (Musanga cecropioides); les grands arbres anthropophiles tels que le fromager (Ceiba pentandra), et l'essesang (Ricinodendron heudelotii); les pieds de Funtumia elastica, avec d'anciennes cicatrices de saignée du fait de leur sève jadis exploitée et utilisée pour la production $\mathrm{du}$ caoutchouc. Toutefois, les fosses archéo-pédologiques permettant de 
retrouver des traces plus anciennes d'occupations et activités humaines (tels les poteries, les charbons de bois...) n'ont pas été réalisées.

\section{Analyse des données}

Les différents indices de diversité floristique classiques ont été calculés pour chaque type de végétation sur la base des données agrégées des parcelles circulaires par type de végétation. Les indices suivants ont été considérés : la richesse spécifique, l'indice de Shannon-Wiener ( $\left.H^{\prime}\right)$, l'indice de Simpson $(D)$, l'Equitabilité de Piélou $(Q)$. Pour le calcul de ces trois derniers indices, le logiciel SPADE (MLE : Minimum Likehood Estimator) (Chao et Shen, 2003), a été utilisé.

L'indice de NNESS a été calculé à partir du programme BiodivR 1.0 (Hardy, 2005), pour évaluer le degré de similarité floristique de différents types de végétation selon la formule: $N N S S E_{\mathrm{ij} / \mathrm{k}}=E S S_{\mathrm{ij} / \mathrm{k}} /\left[\left(E S S_{\mathrm{ij} / \mathrm{k}}+E S S\right.\right.$ $\mathrm{ij} / \mathrm{k}) / 2$ ].

Où, $E S S_{\mathrm{ij} / \mathrm{k}}$ est le nombre d'espèces communes attendues d'un tirage au hasard (sans remplacement) de $k$ individus du relevé $i$ et de $k$ individus du relevé $j$.

La dominance des espèces et des familles a été évaluée par le calcul des surfaces terrières $\left(\mathrm{St}=(1 / 2 \mathrm{~d})^{2} \pi\right.$ où $\left.\mathrm{d}=\mathrm{dhp}\right)$ (Magurran, 1988).

L'analyse multivariée des données d'abondance floristique des différents types de la végétation a été effectuée suivant l'Analyse par Composantes Principales (ACP) ou encore PCA (Principal Components Analysis) à l'aide du logiciel Pc_Ord 4.0 (Mc Cune et Mefford, 1995).

\section{Analyse statistique}

Le logiciel STATISTICA 6.0. (Statistica, 2005) a été utilisé pour les différentes analyses statistiques.

Le test non paramétrique de Wilcoxon a été utilisé pour comparer la distribution des proportions de différents types de végétation identifiés entre les deux sites d'études. Les différences des hauteurs de canopées entre les types de la végétation ont été analysées par le test d'ANOVA à un facteur. Ensuite, le test post hoc de Newman-Keuls a permis la différenciation de groupes homogènes (Siegel et Castellan, 1988).

Le test de Chi-2 a été utilisé pour vérifier si la distribution des réseaux de pistes d'éléphants dans les différents types de la végétation est faite au hasard. Le nombre théorique de réseaux de pistes attendu $(A)$, est calculé comme suit : $A_{i}=\left(x_{i}^{*} y\right) / 100$, où $x_{i}$ est le pourcentage de la végétation de type $i$ et, $y$ le nombre total de réseaux de pistes d'éléphants recensés.

Un indice de préférence de visites des différents types de la végétation $\left(I_{p}\right)$ a été ensuite calculé. $I_{p i}=\left(A_{i}-O_{i}\right) / A_{i}$.

Où $A_{i}$ et $O_{i}$ représentent respectivement le nombre de réseaux de pistes "attendu" et "observé" pour le type de végétation $i$.

\section{RESULTATS}

\section{Faciès de la végétation}

De manière globale pour l'ensemble des deux sites, 11 types de végétations ont été identifiés. Outre les 8 types préalablement définis, une observation minutieuse de la physionomie de la forêt nous a permis de distinguer trois autres types de végétation dont: la forêt secondaire jeune clairsemée (FSJC), elle se rencontre ici en poches peu étendues dans la forêt, la hauteur de sa strate dominante atteignant à peine $5 \mathrm{~m}$ est continue, les arbres qui émergent de la couche dense inextricable du sous-bois apparaissent de manière très éparse et sont de grande taille (pouvant atteindre $25 \mathrm{~m}$ et plus); la végétation touffue, constituée essentiellement d'espèces suffrutescentes, peu étendue et rencontrée principalement sur les berges de certains cours d'eau que nous avons qualifié de fourré galerie (GA) et enfin; les trouées forestières $(\mathrm{TF})$, chablis naturels créés par la chute de grands arbres. 
Le Tableau 1 présente la proportion des différents faciès de la végétation recensés.

Ces chiffres révèlent une prédominance des formations secondarisées aussi bien dans le site situé à l'extérieur de la Réserve (Mimpala), que dans le site situé dans la Réserve (Dingué), soit $52 \%$ et $58 \%$ respectivement. Le test de Wilcoxon, n'a pas montré de différence dans la distribution des proportions des différents types de végétation entre les deux sites $(Z=0,31, p=0,75)$. Toutefois, on note quelques éléments de différence concernant les types peu présents : un pourcentage plus important à la fois des clairières sur sols hydromorphes et des forêts secondaires jeunes clairsemées sur le site à l'intérieur de la Réserve.

\section{Composition floristique et similitude entre les faciès de végétation}

Nous avons recensé au total 1222 individus d'arbres ( $\mathrm{dhp} \geq 10 \mathrm{~cm}$ ) dans les 435 placettes circulaires inventoriées. Un total de 183 espèces appartenant à 38 familles a été inventorié. Les familles les plus représentatives, en terme de nombre d'espèces, sont les Euphorbiacées (30 espèces), les Rubiacées (14 espèces), les Annonacées (13 espèces), les Caesalpiniacées (13 espèces) et les Meliacées (11 espèces).

En terme de nombre d'individus, les familles les plus représentées sont les Euphorbiacées (217 individus dominés par Plagiostyles africana et Uapaca paludosa), Les Apocynacées (123 individus, représentés à $91 \%$ par Tabernaemontana crassa), les Olacacées (112 individus, dominés par Strombosiopsis tetrandra, Heisteria zimmereri et Strombosia pustulata), les Annonacées (108 individus dominés par Greenwayodendron suaveolens; Annikia chlorantha et Anonidium mannii).

En considérant les surfaces terrières, les familles les plus dominantes sont: les Euphorbiacées, les Lecythidacées, les Tiliacées et les Olacacées avec respectivement
$18 \%, 11 \%, 8 \%$ et $6 \%$ des surfaces basales totales. Les espèces ayant une forte dominance sont par ordre décroissant des surfaces terrières à l'hectare: Petersianthus macrocarpus $\quad\left(2,9 \quad \mathrm{~m}^{2} / \mathrm{ha}\right), \quad$ Duboscia macrocarpa $\left(2 \mathrm{~m}^{2} / \mathrm{ha}\right)$, Uapaca paludosa $(1,9$ $\left.\mathrm{m}^{2} / \mathrm{ha}\right)$, Desbordesia glaucescens $\left(0,9 \mathrm{~m}^{2} / \mathrm{ha}\right)$. Les arbres de diamètre $\leq$ à $20 \mathrm{~cm}$ constituent $53 \%$ de tous les individus.

Les indices de diversité de Shannon sont respectivement de 5,5 et 5,4 respectivement pour les sites 1 et 2 . Le Tableau 2 donne les valeurs des différents indices de diversités dans les deux sites.

Le calcul de l'indice de similitude de NNESS entre les sites de Mimpala et de Dengué montre une similarité supérieure à $60 \%$. Cette forte similarité se confirme aussi bien pour les petites que pour les grandes valeurs de $\mathrm{k}\left(\mathrm{NNSES}_{\mathrm{k}=20}=0,63 ; \mathrm{NNSES}_{\mathrm{k}=100}\right.$ $=0,65)$. Nous avons ainsi pu, à la suite des analyses, considérer l'ensemble des deux sites en un seul tenant.

Le coefficient de similarité de NNESS ne permet pas de différencier les faciès de forêt dense mature (FDM), forêt dense secondaire âgée (FDSA) et forêt dense secondaire (FDS). Les indices de similitude de NNESS obtenus sont supérieurs à 50\% aussi bien en considérant les petites que les grandes valeurs $\mathrm{k}$. Le calcul de cet indice est essentiellement basé sur la composition floristique. Les autres critères liés à la structure de la végétation, notamment la hauteur de la canopée dominante, que nous avons pris en compte lors de la caractérisation sur le terrain, nous ont permis de faire une différenciation de ces faciès. L'analyse de la variance des hauteurs de la canopée a permis de noter qu'il existe une nette différenciation entre les différents faciès de la végétation (ANOVA, ddl=9; $F=69, \mathrm{p}<0,001$ ). Le test de Newman-keuls a ensuite permis de différencier des groupes homogènes (Figure 2). 
La carte d'analyse factorielle obtenue après la mise en commun des données de la composition et de l'abondance floristique de chaque faciès de la végétation révèle une opposition entre les formations de forêts secondaires jeunes et de forêts denses matures (Figure 3). L'isolement des forêts ripicoles et des raphiales marque la forte influence du facteur édaphique lié au caractère hydromorphe du sol. L'axe 1 et 2 contribuent à expliquer respectivement à $17 \%$ et $10 \%$ la variance observée entre les différents faciès.

\section{Actions des éléphants}

L'action des éléphants est très marquée dans les deux sites, en moyenne 2,68 réseaux de pistes/km ont été recensés dans le site 1 (Mimpala) et 3,74/km dans le site 2 (Dengué). Dans la forêt ripicole, la forêt marécageuse et les clairières, les empreintes sont denses et omniprésentes. Ces milieux ne se prêtent pas au dénombrement des pistes d'éléphants et n'ont donc pas été pris en compte dans l'estimation.

Les réseaux de pistes d'éléphants recensés ne présentent pas une distribution au hasard au sein de la végétation $\left(\mathrm{ddl}=6 ; \chi^{2}=\right.$ $34 ; \mathrm{p}<0,01)$. En considérant l'indice de préférence de visite, on remarque qu'ils ont une préférence pour les formations forestières jeunes dont les anciennes pistes forestières et les trouées forestières (Figure 4). 194 trouées naturelles ont été en tout recensées, avec environ 2 trouées $/ \mathrm{km}$. Dans $35 \%$ des cas, ces trouées ont été perturbées par les éléphants (réseaux de pistes, empreintes, arbres cassés/écorcés). Ils contribuent ainsi par leurs actions à agrandir ou à entretenir ces trouées.

Le faible indice de préférence observé pour les forêts secondaires jeunes "clairsemées" est contradictoire aux résultats auxquels on se serait attendus. En effet, ce faciès de la végétation constitue une niche alimentaire importante pour les éléphants. Ces derniers l'utilisent pour leur alimentation et donc n'y développent pas nécessairement de réseaux permanents de pistes.

\section{Marques visibles d'anciennes présences humaines dans la forêt}

Plus d'une soixantaine de marques visibles de l'action de l'homme dans un passé plus ou moins récent ont été recensées. Les ngùla, et les taches de grands palmiers étaient les plus présents, avec respectivement $43 \%$ et $28 \%$ des indices recensés. En dehors des formations de forêts secondaires, ces marques n’ont pas été recensées dans les autres types de végétation (Tableau 3).

Tableau 1: proportion* des différents faciès de la végétation.

\begin{tabular}{lccc}
\hline Faciès de la végétation & Site Mimpala & Site Dengué & Sites 1 et 2 \\
\hline Forêt dense Mature (FDM) & $17 \%$ & $14 \%$ & $14 \%$ \\
Forêt dense secondaire âgée (FDSA) & $23 \%$ & $24 \%$ & $24 \%$ \\
Forêt dense secondaire (FDS) & $23 \%$ & $15 \%$ & $19 \%$ \\
Forêt secondaire jeune (FSJ) & $5 \%$ & $10 \%$ & $7 \%$ \\
Forêt secondaire jeune clairsemée (FSJC) & $1 \%$ & $9 \%$ & $5 \%$ \\
Forêt ripicole (RIP) & $7 \%$ & $10 \%$ & $9 \%$ \\
Raphiale (RAP) & $11 \%$ & $9 \%$ & $8 \%$ \\
Fourré galerie (GA) & $1 \%$ & $2 \%$ & $1 \%$ \\
Clairière (Prairie marécageuse) (CL) & $0,10 \%$ & $2 \%$ & $1 \%$ \\
Piste forestière (PI) & $2 \%$ & $0 \%$ & $1 \%$ \\
Trouée forestière (TF) & $13 \%$ & $7 \%$ & $10 \%$ \\
$*$ La proportion de chaque type de végétation est obtenue en faisant le rapport entre la distance couverte par végétation \\
considérée et la distance totale de l'ensemble des transects.
\end{tabular}


G. M. NGUENANG et al. / Int. J. Biol. Chem. Sci. 4(5): 1766-1781, 2010

Tableau 2 : Indices de diversité des différents types de végétation de la forêt du Dja.

\begin{tabular}{lccccccccccc}
\hline & Site1 & \multicolumn{1}{c}{ Mimpala } \\
\hline & & FDM & FDSA & FDS & FSJ & FSJC & GA & RA & RIP & TF & PI \\
\hline Nombre de relevés circulaires & $\mathbf{2 2 5}$ & 33 & 40 & 50 & 6 & 16 & 3 & 26 & 17 & 31 & 3 \\
Nombre d'espèces recensées & $\mathbf{1 3 2}$ & 59 & 61 & 64 & 18 & 24 & 6 & 8 & 29 & 38 & 9 \\
Nombre d'individus recenses & $\mathbf{6 1 7}$ & 115 & 134 & 164 & 26 & 38 & 6 & 12 & 43 & 48 & 10 \\
L'indice de Shannon-Wiener (H') & $\mathbf{5 , 5}$ & 3,72 & 3,89 & 3,67 & 2,67 & 3,07 & 1,61 & 2,27 & 3,34 & 3,523 & 2,16 \\
Indice de Simpson (D) & $\mathbf{0 , 0 1}$ & 0,03 & 0,03 & 0,04 & 0,09 & 0,06 & 0,2 & 0,11 & 0,04 & 0,04 & 0,12 \\
Equitabilité de Piélou (Q) & $\mathbf{0 , 8 3}$ & 0,79 & 0,81 & 0,77 & 0,74 & 0,71 & 0,7 & 0,76 & 0,8 & 0,81 & 0,75 \\
& Site 2 & & & & & & & & Dengue & & \\
Nombre de relevés circulaires & $\mathbf{2 1 0}$ & 26 & 42 & 42 & 16 & 18 & 5 & 17 & 22 & 22 & \\
Nombre d'espèces recensées & $\mathbf{1 4 5}$ & 55 & 69 & 65 & 25 & 26 & 10 & 13 & 39 & 33 \\
Nombre d'individus recenses & $\mathbf{6 0 5}$ & 98 & 145 & 144 & 43 & 35 & 9 & 15 & 70 & 46 \\
L'indice de Shannon-Wiener (H') & $\mathbf{5 , 4}$ & 3,85 & 3,75 & 3,67 & 2,89 & 2,83 & 2,04 & 2,15 & 3,34 & 3,52 \\
Indice de Simpson (D) & $\mathbf{0 , 0 1}$ & 0,03 & 0,04 & 0,05 & 0,07 & 0,09 & 0,14 & 0,14 & 0,05 & 0,04 \\
Equitabilité de Piélou (Q) & $\mathbf{0 , 8 4}$ & 0,82 & 0,77 & 0,76 & 0,77 & 0,75 & 0,71 & 0,72 & 0,77 & 0,78 \\
\hline
\end{tabular}

FDM : Forêt dense mature ; FDSA : Forêt dense secondaire âgée ; FDS : Forêt dense secondaire ; FSJ : Forêt secondaire jeune ; FSJC : Forêt secondaire jeune clairsemée; RIP : Forêt ripicole ; RAP : Raphiale ; GA : Fourré galerie ; CL : Clairière (Prairie marécageuse) ; PI : Piste forestière ; TF : Trouée forestière

Tableau 3: Marques d'anciennes présences humaines dans la forêt.

\begin{tabular}{|c|c|c|c|c|c|}
\hline \multirow{2}{*}{ Végétation } & \multicolumn{3}{|c|}{ Indices d'anciens Sites } & \multirow{2}{*}{\multicolumn{2}{|c|}{ Autres $^{(i i)}$ Total }} \\
\hline & Ngùla $^{(i)}$ & Taches grands palmiers & Parasoleraies & & \\
\hline Forêt dense secondaire & 13 & 10 & 1 & 9 & 33 \\
\hline Forêt dense secondaire âgée & 5 & 2 & 0 & 2 & 9 \\
\hline Forêt secondaire jeune & 8 & 2 & 2 & 3 & 15 \\
\hline Forêt secondaire jeune clairsemée & 6 & 7 & 2 & 2 & 17 \\
\hline Total & 32 & 21 & 5 & 16 & 74 \\
\hline
\end{tabular}


G. M. NGUENANG et al. / Int. J. Biol. Chem. Sci. 4(5): 1766-1781, 2010

Tableau 4 : Proportions des différents types de forêts obtenues par différents auteurs dans le Dja, les autres sites d'étude, et au niveau national au Cameroun.

\begin{tabular}{|c|c|c|c|}
\hline Référence & Types de forêts & Composantes & Proportion \\
\hline \multirow[t]{3}{*}{ Sonke (1996) (forêt du Dja, Est Cameroun) } & Forêts de terre ferme & $\begin{array}{l}\text { Forêt hétérogène typique ; Forêt } \\
\text { primaire à rotangs ; Forêt à } \\
\text { Gilbertiodendron ; Forêt à raphia } \\
\text { regalis }\end{array}$ & $75 \%$ \\
\hline & $\begin{array}{l}\text { Forêts sur sols } \\
\text { hydromorphes }\end{array}$ & & $20 \%$ \\
\hline & Forêts sur rocher & & $5 \%$ \\
\hline \multirow{5}{*}{ Mbolo (2004) (forêt du Dja, Est Cameroun) } & Forêt primaire & & $84 \%$ \\
\hline & Forêts secondaires & & $7,5 \%$ \\
\hline & Jachères et cultures & & \\
\hline & $\begin{array}{l}\text { Forêt à Gilbertiodendron } \\
\text { Forêts sur sols }\end{array}$ & & $<$ à $0,5 \%$ \\
\hline & hydromorphes & & $6 \%$ \\
\hline \multirow{3}{*}{ Dupain et al. (2004) (périphérie nord du Dja, Est Cameroun) } & Forêt primaire & & $17,6 \%$ \\
\hline & Forêts secondaires & $\begin{array}{l}\text { Forêt secondaire âgée }(10,8 \%) \text {, Forêt } \\
\text { secondaire }(39,3 \%) \text {, Jeune forêt } \\
\text { secondaire }(15,6 \%)\end{array}$ & $65,8 \%$ \\
\hline & $\begin{array}{l}\text { Forêts sur sol } \\
\text { hydromorphe }\end{array}$ & & $9,3 \%$ \\
\hline Fongnzossie et al. (2008) (Mégamé, Sud Cameroun) & Forêts secondaires & & $54 \%$ \\
\hline Van Germerden et al. (2003) (Akom II-Bipindi ; Sud Cameroun) & Forêts secondaires & & $40-60 \%$ \\
\hline \multirow{3}{*}{ Présente étude (forêt du Dja, Est Cameroun) } & Forêt mature & & $14 \%$ \\
\hline & Forêts secondaires & $\begin{array}{l}\text { Forêt secondaire âgée }(24 \%) \\
\text { Forêt dense secondaire }(19 \%) \text {, Forêt } \\
\text { secondaire jeune }(7 \%) \text {, Forêt } \\
\text { secondaire jeune clairsemée }(5 \%)\end{array}$ & $55 \%$ \\
\hline & $\begin{array}{l}\text { Forêts sur sols } \\
\text { hydromorphes }\end{array}$ & $\begin{array}{l}\text { Forêt ripicole ; Raphiale ; Fourrée } \\
\text { galerie, Prairie marécageuse }\end{array}$ & $19 \%$ \\
\hline
\end{tabular}




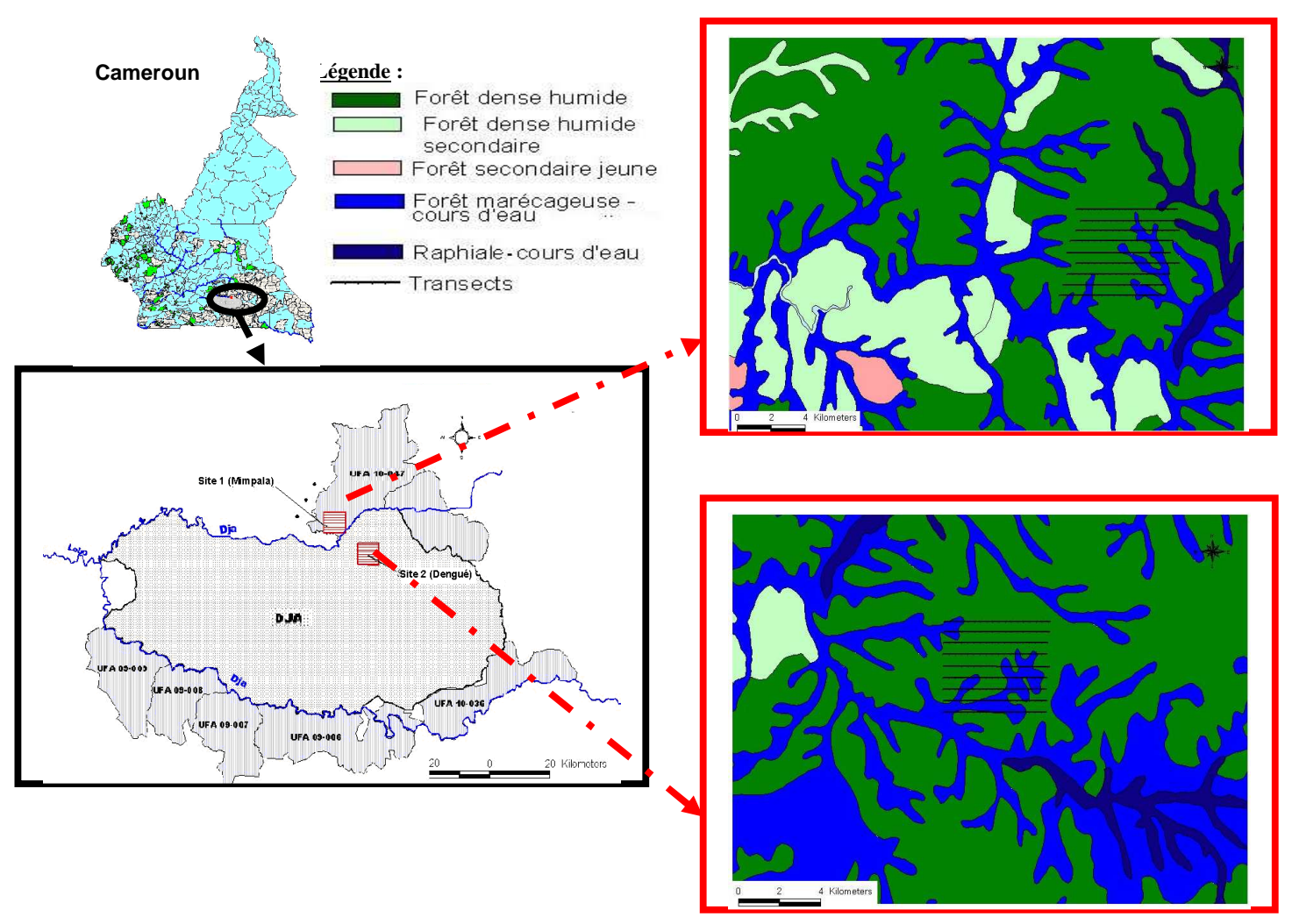

Figure 1 : Zone d'étude et carte de la végétation des sites d'étude (d'après WRI -GFW, 2005).

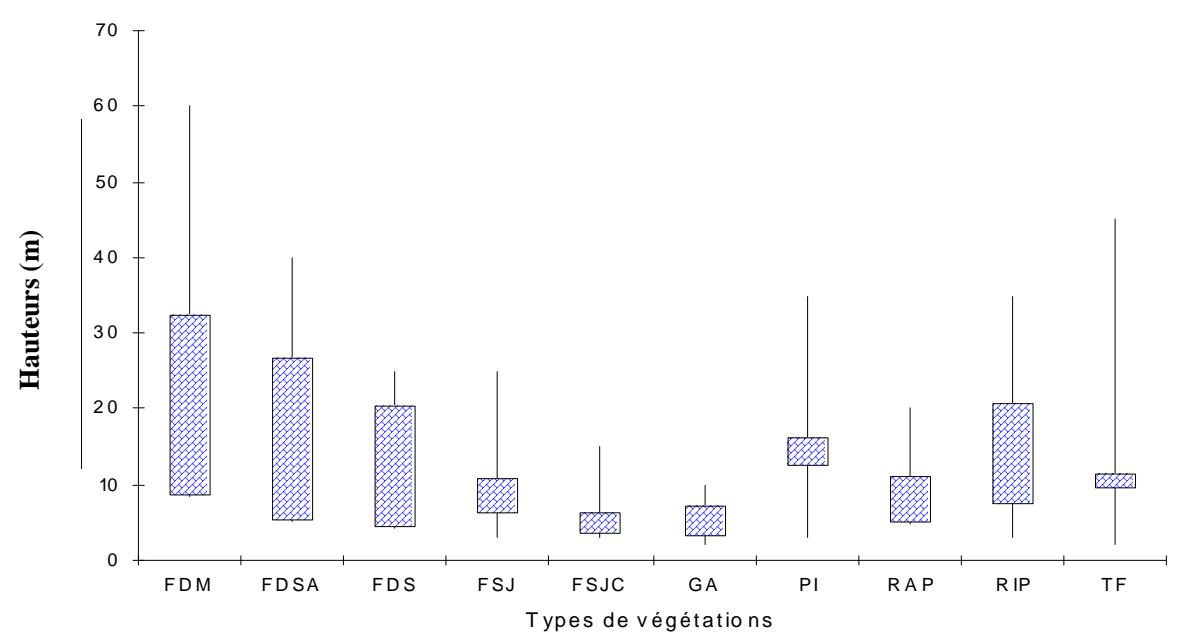

Figure 2 : Distribution de la hauteur de la strate dominante dans les différents faciès de la végétation.

(i) : les lettres différentes indiquent les différences significatives.

FDM : Forêt dense mature ; FDSA : Forêt dense secondaire âgée ; FDS : Forêt dense secondaire ; FSJ : Forêt secondaire jeune ; FSJC : Forêt secondaire jeune clairsemée; RIP : Forêt ripicole ; RAP : Raphiale ; GA : Fourré galerie ; CL : Clairière (Prairie marécageuse) ; PI : Piste forestière ; TF : Trouée forestière. 


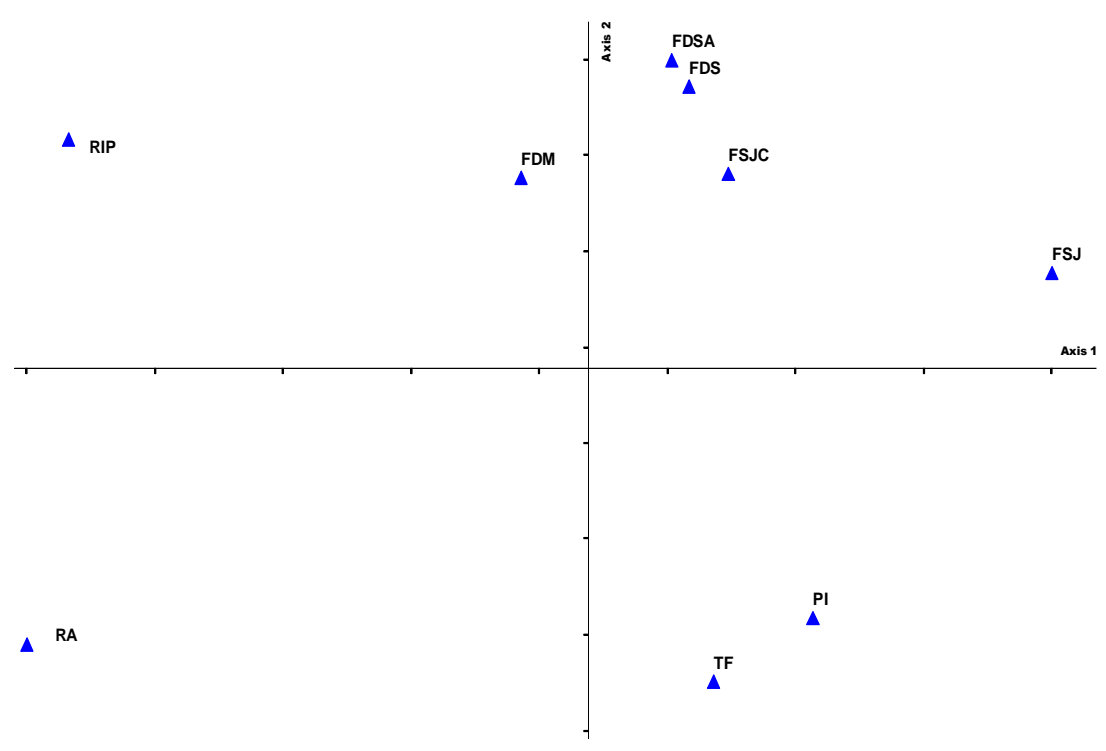

Figure 3 : Carte d'analyse factorielle (PCA) des différents faciès de la végétation basée sur l'abondance et la composition floristique des deux sites (Mimpala et Dingué).

FDM : Forêt dense mature; FDSA : Forêt dense secondaire âgée; FDS : Forêt dense secondaire; FSJ : Forêt secondaire jeune; FSJC : Forêt secondaire jeune clairsemée; RIP : Forêt ripicole ; RAP : Raphiale ; GA : Fourré galerie ; CL : Clairière (Prairie marécageuse) ; PI : Piste forestière ; TF : Trouée forestière.

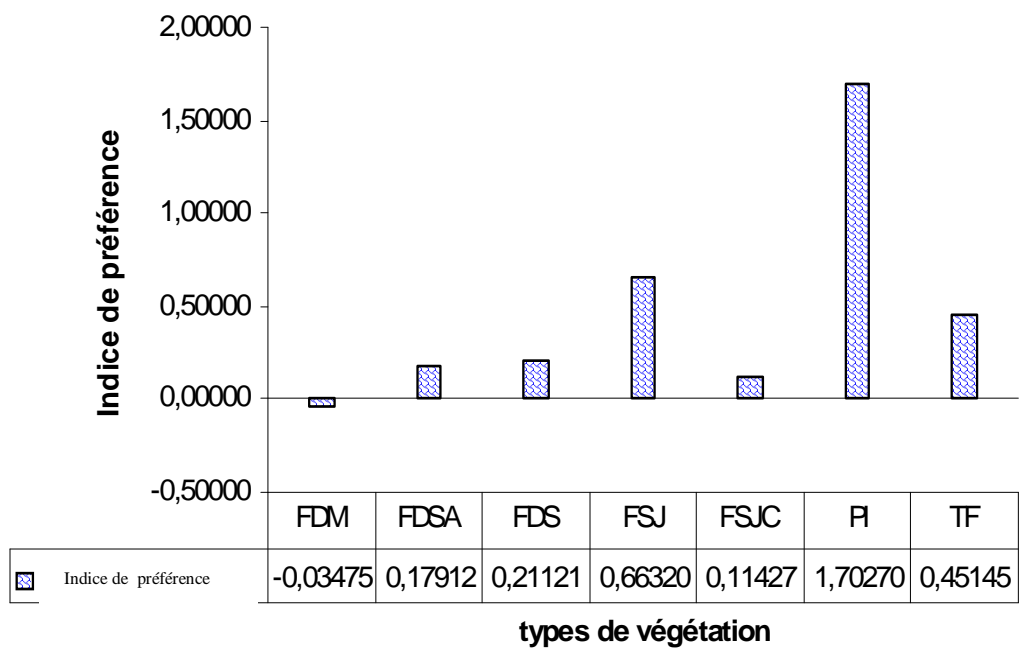

Figure 4 : Indices de préférence de visite des différents types de la végétation de la forêt de terre ferme par les éléphants, sur base de la densité des réseaux de pistes (cf. méthodologie pour les détails).

FDM : Forêt dense mature; FDSA : Forêt dense secondaire âgée ; FDS : Forêt dense secondaire ; FSJ : Forêt secondaire jeune ; FSJC : Forêt secondaire jeune clairsemée; RIP : Forêt ripicole ; RAP : Raphiale ; GA : Fourré galerie ; CL : Clairière (Prairie marécageuse) ; PI : Piste forestière ; TF : Trouée forestière. 


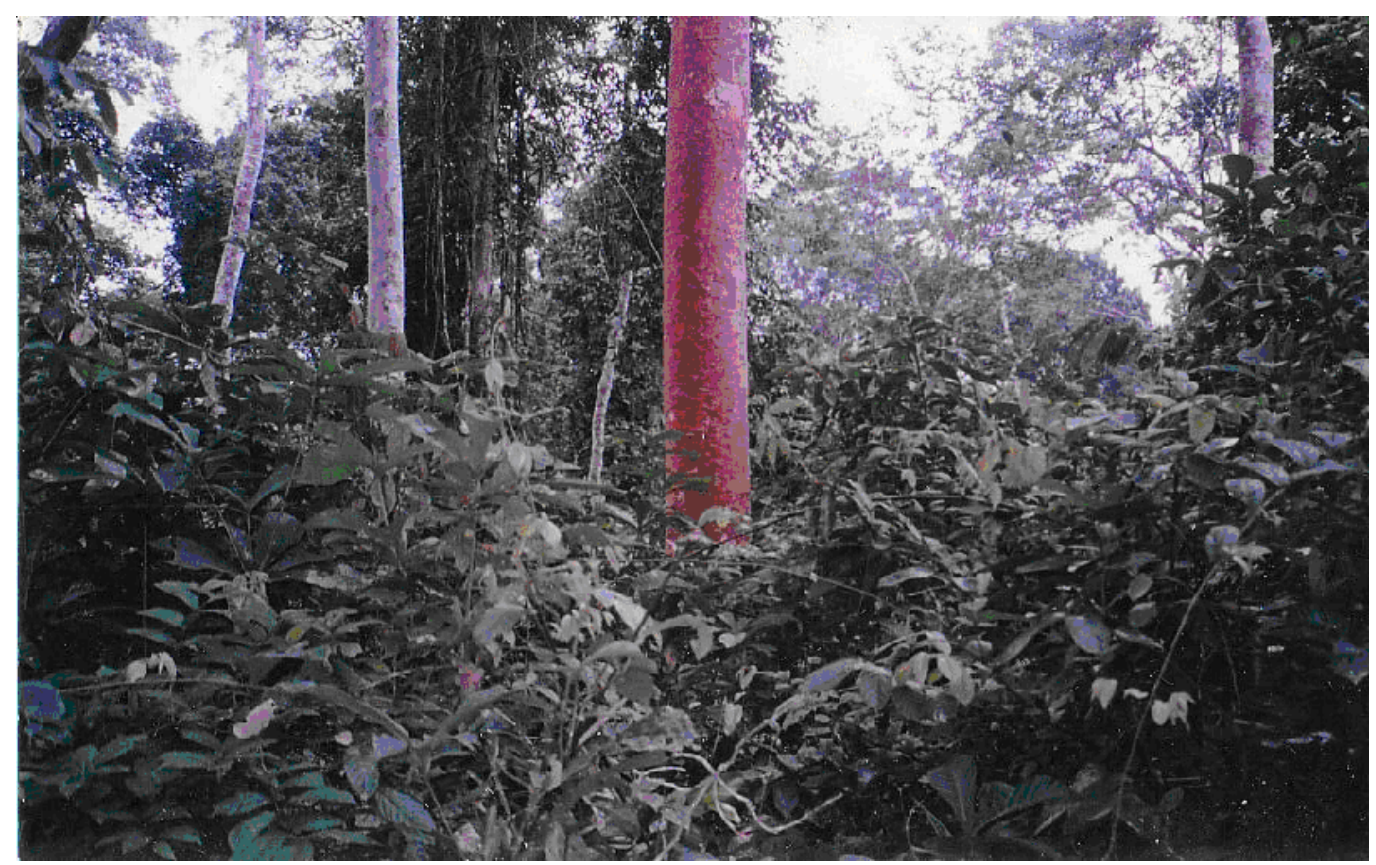

Figure 5 : Poche de forêt clairsemée issue de l'action des éléphants sur la forêt secondaire jeune. "On note un décalage net entre la strate herbacée et la strate ligneuses assez éparse"

\section{DISCUSSION}

Cette étude confirme la grande proportion de forêts secondaires dans le massif forestier camerounais (Tableau 4). Par contre, elle semble contradictoire aux résultats passés obtenus par Mbolo (2004). Ces différences observées, pourraient s'expliquer par la définition faite par les uns et les autres de la notion de forêt secondaire. La définition accordée dans cette étude à la formation secondaire, ne se limite pas aux vieilles jachères préforestières encore fortement marquées par les espèces pionnières comme Musanga cecropioides. Nous avons distingué les jeunes forêts secondaires très caractéristiques et les forêts denses secondaires âgées. En effet, même si après plusieurs décennies, il s'est formé une sylve ayant l'aspect de la forêt "climacique", quelques grands arbres héliophiles longévifs, la forte densité de la strate inférieure et la structure de la canopée, permettent de différencier ces formations secondaires âgées (Emrich et al., 2000 ; Van Gemerden et al., 2003).
Mayaux et al. (2001) ont évalué par télédétection spatiale, que les forêts secondaires représentent seulement $8 \% \mathrm{du}$ couvert forestier camerounais et $8,27 \%$ pour l'ensemble de la forêt du bassin du congo. Ces auteurs font toutefois remarquer que cette méthode basée sur la photo-interprétation et l'analyse d'images satellitaires, bien qu'efficace pour l'observation de l'évolution du couvert forestier au niveau régional, peut présenter des limites dans la caractérisation de la végétation à un niveau local.

Bien que notre étude soit localement limitée et ne couvre pas toute la zone de la forêt du Dja, nos résultats se rapprochent de ceux obtenus par certains auteurs dans d'autres sites forestiers au Cameroun, même si les méthodologies utilisées ne sont pas tout à fait similaires (Van Gemerden et al., 2003; Dupain et al., 2004 ; Fongnzossie et al., 2008).

Tchouto (2004), note que la forêt côtière de Campo-Ma'an à l'extrême Sudouest du Cameroun, révèle de part sa composition floristique actuelle, les marques de sa forte perturbation anthropique passée; 
les poches de forêts "primaires" peu ou pas perturbées se trouvant seulement sur les sommets de colline ou les pentes difficiles d'accès.

Au regard des proportions des forêts secondaires observées, il importe de s'attarder sur les origines et les causes de la secondarisation de la forêt tropicale. Plusieurs auteurs ont décrit le processus de secondarisation de la forêt tropicale (Nkongmeneck, 1998 ; Emrich et al., 2000). Nous reprendrons ici les principaux facteurs relevés, en insistant ici sur les causes liées aux perturbations anthropiques dans un passé récent, à l'action des éléphants et aux phénomènes paléo-environnementaux.

\section{Actions passées de l'homme sur la végétation \\ Plusieurs études archéologiques et} anthracologiques ont contribué à montrer l'influence de l'homme sur les forêts tropicales. Clist (1990) fait remarquer que la plupart des chercheurs, peu au fait des connaissances archéologiques, traitent les zones forestières tropicales africaines comme des forêts vierges. Les recherches archéologiques récentes démontrent de manière concordante, la présence des chasseurs-cueilleurs avant, pendant et après l'arrivée des sociétés sédentaires vers 5000 ans BP (Clist, 1990).

Schwartz (1992) situe l'implantation des populations bantoues dans le grand bloc forestier d'Afrique centrale aux alentours de 3000 et 2000 ans BP.

La prospection menée dans la partie Sud-est du Dja par Ossah Mvondo (1993) a permis de mettre en évidence des sites d'occupation préhistorique et métallurgique ainsi que des zones de peuplement ancien.

Bien que Vansina (1991) ait démontré le caractère rudimentaire de l'agriculture par le passé, du fait du peu d'intérêt, de temps accordé à celle-ci, la grande mobilité des peuples de forêt a contribué à perturber l'ensemble $\mathrm{du}$ paysage forestier. Nkongmeneck (1998) insiste sur les perturbations liées aux déplacements successifs des campements des chasseurs, de pêcheurs, et de cueilleurs qui affectent notamment le sous-bois. Aussi, Mbida et al. (2001) ont mis en évidence l'expansion de l'agriculture en zone forestière camerounaise, avec notamment, l'introduction de la culture de la banane il y a environ 2500 ans BP.

Vermeulen (2000), a identifié dans le Dja plusieurs sites d'anciens villages datant d'un peu plus d'un demi-siècle à plus de deux siècles.

\section{Phénomènes paléo-environnementaux}

La présence humaine antérieure ne peut être seule mise en cause pour expliquer l'aspect en mosaïque et l'importante secondarisation des forêts.

Les formations végétales sous les tropiques ont subi d'importantes variations climatiques au cours du quaternaire. Les destructions catastrophiques des forêts d'Afrique centrale survenues il y'a environ 2500 ans exercent encore une influence majeure sur la répartition actuelle des formations végétales (Maley, 2001).

La grande partie de l'Afrique centrale et de l'ouest a été affectée par une phase de réchauffement climatique entre 3000 et 2000 ans BP. Au cours de celle-ci, les formations forestières se sont fragmentées en favorisant les espaces plus ou moins ouverts, repris par les hommes qui ont commencé à développer les premières plantations de palmiers à huile (Maley, 1992 ; Schwartz, 1992 ; ReynaudFerrera et al., 1996 ; Maley et Brenac, 1998 ; Ngomanda et al., 2009).

Dans la forêt du Dja, les périodes de péjorations climatiques auraient engendré une fragmentation de la forêt qui aurait permis une expansion dans la région des espèces pionnières et héliophiles telles que : Tabernaemontana crassa, Alstonia boonei, Petersianthus macrocarpus, Terminalia superba et Triplochiton scleroxylon. Les études palynologiques restent toutefois nécessaires pour vérifier cette hypothèse.

Les faciès de forêts denses de type "climacique" rencontrés ici, particulièrement sur les versants des collines près des rivières, 
constitueraient des micro-refuges. Senterre (2005) faisait déjà remarquer que les divers " refuges " qui ont pu subsister n'ont pas toujours été constitués de blocs forestiers d'un seul tenant mais aussi de mosaïques de microrefuges formés par des collines isolées, des forêts-galeries, des versants bien exposés, etc.

\section{Actions des éléphants}

Les éléphants, bien que jouant un rôle important dans la dynamique de régénération forestière par la dissémination des diaspores, contribuent aussi à la perturbation de la végétation. D'après Hemborg et al. (2006), les éléphants peuvent sérieusement endommager voire tuer les arbres en prélevant une part de la cime, de l'écorce ou en cassant le tronc. Comme celles de Plumtre (1993), nos données montrent que la perturbation des éléphants dépend du type de forêt. Les milieux déjà perturbés et ouverts sont préférentiellement visités par les éléphants (Vanleeuwe et Gautier, 1998 ; Blake et Inkamba-Nkulu, 2004 ; Paul et al., 2004). Dans le Dja, l'intense activité des éléphants dans les jeunes forêts secondaires est à l'origine d'un faciès particulier de la forêt que nous avons qualifié de "forêt secondaire jeune clairsemée". Cette forêt présente une physionomie très similaire à celle des forêts clairsemées à Marantaceés rencontrées à l'extrême Sud-est du Cameroun et au Nord du Congo (Figure 5). Alors que ces dernières tireraient leur origine des changements climatiques importants observés les cent derniers mille ans au Pléistocène (White et Oslisly, 1998), les poches de forêt secondaires jeunes clairsemées rencontrées ici résulteraient principalement de l'action combinée de l'homme et des éléphants sur la végétation. Ces poches peu étendues dans la forêt constitueraient probablement d'après les marques qui y ont été recensées et des informations obtenues des villageois, des sites d'anciens villages abandonnés. Les éléphants semblent contribuer à maintenir les formations secondaires à un stade jeune, au travers des visites fréquentes de ces formations et notamment du piétinement qui affecte considérablement la croissance des plantules (Campbell et al., 1996).

L'exploitation forestière et l'agriculture sont souvent accusées d'être la cause essentielle de dégradation de la forêt. S'il est certes vrai qu'elles y jouent un rôle important, la secondarisation de la forêt dense d'Afrique centrale telle que observée aujourd'hui découle aussi d'autres facteurs notamment des actions anthropiques traditionnelles passées. Notre étude permet de montrer que la proportion des forêts secondaires est similaire dans le site situé hors de la Réserve que celui situé dans la Réserve et n'ayant jamais fait l'objet d'une exploitation forestière industrielle. Les faciès de jeunes formations secondaires constituent les pôles d'attractions pour les éléphants et bien d'autres espèces phares comme les gorilles et les chimpanzés (Dupain et al., 2004). Aussi ces faciès constituent des habitats préférentiels pour la nidification des grands singes (Nguenang et al., 2005).

Dans le Dja les jeunes formations secondaires clairsemées pourraient avoir une forte implication pour la conservation. En effet l'identification de ces faciès de jeunes formations secondaires très caractéristiques et les sites d'anciens villages pourrait être intégrée dans les stratégies d'aménagement des aires protégées et la définition des séries de conservation au sein des concessions forestières. Ils devraient également être pris en compte comme un élément relevant pour l'identification des forêts à haute valeur de conservations dans le cadre des critères exigibles pour la certification forestière, concept actuellement promu afin de garantir la gestion durable et équitable des forêts.

\section{REMERCIEMENTS}

Cette étude a été menée dans le cadre de la collaboration entre Laboratoire de Systématique et d'Ecologie végétales de l'Université de Yaoundé I et le "Projet Grands Singes" du Jardin zoologique d'Anvers. Nous tenons à remercier ce Projet qui nous a accordé toutes les facilités logistiques de terrain pour mener cette étude. Nous avons eu 
des échanges constructifs avec Gilles Dauby du Laboratoire d' Eco-Ethologie Evolutive de l'Université Libre de Bruxelles (Belgique). Nous remercions Sylvie Bekah, Nadine De Jong qui ont participé activement à la collecte des données. Nous ne saurons oublier les communautés des villages Malen V, Doumopierre, Mimpala, et Akom qui nous ont favorablement accueilli et ont permis d'effectuer le travail de terrain dans de très bonnes conditions.

\section{REFERENCES}

Blake S, Inkamba-Nkulu C. 2004. Fruit, minerals, and forest elephant trails: do all roads leads to Rome? Biotopica, 36(3): 392-401.

Ben yahmed D, Houstin N, Seignobos C. 2005. Atlas du Cameroun. Jeune Afrique : Paris.

Campbell BM, Butler JRA, Mapaure I, Vermeulen SJ, Mashove P. 1996. Elephant damage and safari hunting in Pterocarpus angolensis woodland in northwestern Matabeleland, Zimbabwe. Af. J. Ecology, 34: 380-388.

Chao A, Shen T-J. 2003. Program SPADE (Species Prediction and Diversity Estimation). Program and User's Guide published at http://chao.stat.nthu.edu.tw.

Clist R. 1990. Des derniers chasseurs aux premiers métallurgistes : sédentarisation et début de la métallurgie du fer (Cameroun, Gabon, Guinée équatoriale). In Paysage Quaternaire de l'Afrique Centrale Atlantique, Lanfranchi R, Schwartz D (eds). ORSTOM: Paris; 458478.

Dupain J, Guislain P, Nguenang GM, De Vleeschouwer K, Van Elsacker L. 2004. High chimpanzee and gorilla densities in a non-protected area on the northern periphery of the Dja Faunal Reserve, Cameroon. Oryx, 38(2): 209-215.

Emrich A, Pokorny B, Sepp C. 2000. The Significance of Secondary Forest Management for Development Policy. GTZ/TÖB/ECO: Eschbonn.
Fongnzossie FE, Tsabang N, Nkongmeneck BA, Nguenang GM, Auzel P, Ellis C, Kamou E, Balouma JM, Apalo P, Halford M, Valbuena M, Valere M. 2008. Les peuplements d'arbres du Sanctuaire à gorilles de Mengamé au sud Cameroun. Tropical Conservation Science, 3: 204221.

Genot J-C. 2006. Vers un changement «climacique»? Courrier de l'Environnement INRA, 53: 129-131.

Hardy O. 2005. BiodivR 1.0. A Program to Compute Statistically Unbiased Indice of Species Diversity Within Samples and Species Similarity Between Samples using Rarefaction Principles. User's Manuel, Université Libre de Bruxelles: Belgique.

Hart TB, Hart JA, Deschamps R, Fournier M, Atoholo M. 1996. Changes in forest composition over the last 4000 years in the Ituri bassin, Zaire. In The Biodiversity of African Plants, Van Der Maesen LJG, Van Der Burgt XM, Van Der Medenbach De Rooy JM (eds). Doredrecht, Kluwer Academic: The Netherlands; 545-563.

Hembourg AM, Bond JW. 2006. Do browsing elephants damage female trees more? Af. J. Ecology, 45: 41-48.

Lejoly J. 1995. Suivi des programmes botaniques et ethnobotaniques dans la Réserve de Faune du Dja. Projet ECOFAC, AGRECO-CTFT, Yaoundé.

Letouzey R. 1985. Notice de la carte phytogéographique du Cameroun au 1/500.000, Institut de la carte internationale de la végétation, Toulouse, France.

Magurran AE. 1988. Ecological Diversity and its Measurement. Croom Helm : London.

Maley J, Brenac P. 1998. Les variations de la végétation et des paleoenvironnements du sud Cameroun au cours des derniers millénaires. Etude de l'expansion du palmier a huile. In Geosciences au Cameroun. Vicat JP, Bilong P (eds). Geocam $\mathrm{n}^{\circ} 1$, Presses Universitaire : Cameroun, Yaoundé ; 85-97.

Maley J. 1992. Mise en évidence d'une péjoration climatique entre ca. 2500 et 
2000 ans BP en Afrique tropicale humide. Bull. Soc. Géol. France, 163: 363-365.

Maley J. 2001. La destruction catastrophique des forêts d'Afrique centrale survenue il y'a environ 2500 ans exerce encore une influence majeure sur la répartition actuelle des formations végétales. National Botanic Garden Belgium. Syst. Geogr., 71: 777-796.

Mayaux P, Malingreau J-P. 2001. Couvert forestier d'Afrique centrale : un nouvel état des lieux. Bulletin des Séances Académiques de la Société Royale d'Outre-Mer, 46(2000-4): 475-480.

Mbida CM, Doutrelepont H, Vrydaghs L, Swennen RL, Wennen RJ, Beeckman H, De Langhe E, De Maret P. 2001. First archaeological evidence of banana cultivation in central Africa during the third millennium before present. Vegetation History and Archeolobotany, 10:1-6.

Mbolo M. 2004. Cartographie et typologie de la végétation de la Réserve de la biosphère du Dja. Thèse doctorat d'Etat, Université de Yaoundé 1, p. 144.

Mc Cune B, Mefford MJ. 1995. PC-ORD. Multivariate Analysis of Ecological Data. Version 2.0. Gleneden Beach, Oregon: USA.

Ngomanda A, Neumann K, Schweizer A, Maley J. 2009. Seasonality change and the third millennium BP rainforest crisis in southern Cameroon (Central Africa). Quaternary Research, 71: 307-318.

Nguenang GM, Dupain J, Nkongmeneck BA. 2005. Perturbation de la végétation par les éléphants et distribution spatiale de gorilles de plaine de la forêt du Dja. Parc et Réserve, 60(3): 22-27.

Nkongmeneck A. 1998. Processus de secondarisation en forêt dense humide camerounaise. In La Gestion des Forêts Denses Africaines Aujourd'hui, Nasi R, Amsallem I, Drouineau S (eds). CDROM, FORAFRI : Libreville, Gabon.

Oslisly R. 2006. Les traditions culturelles de l'Holocène sur le littoral du Cameroun entre Kribi et Campo. In Grundlegungen.
Beiträge zur Europäischen und Afrikanischen Archäologie für, Manfred KH, Eggert Tübingen, Wotzka HP (eds). Francke Attempto Verlag $\mathrm{GmbH}$ and Co. KG: Tübingen; 303-317.

Oslisly R, Pickford M, Dechamps R, Fontugne M, Maley J. 1994. Sur une présence humaine mi-Holocène à caractère rituel en grottes au Gabon. Comptes Rendus de l'Académie des Sciences de Paris, 319(II): 1423-1428.

Ossah Mvondo JP. 1993. Prospection des sites d'habitat dans les arrondissements de Djoum et Mintom (Sud Cameroun). Nyame Akuma, 39: 15-16.

Paul JR, Randle AM, Champan CA, Chapman LJ. 2004. Arrested succession in logging gaps: is tree seedling growth and survival limiting? Afr. J. Ecol., 42: 245-251.

Plumtre AJ. 1993. The effects of trampling damage by herbivores on the vegetation of the Parc National des Volcans, Rwanda. Af. J. Ecology, 32: 115-139.

Reynaud-Ferrera I, Maley J, Wirrmann D. 1996. Végétation et climat dans les forêts du sud-ouest Cameroun depuis 4770 ans BP : analyse pollinique des sédiments du lac Ossa. C.R. Acad. Sci., 322(IIa): 749755.

Schwartz D. 1992. Assèchement climatique vers 3000 B.P. et expansion Bantu en Afrique Centrale Atlantique: Quelques réflexions. Bull. Soc. Géol. France, 163: 353-361.

Senterre B. 2005. Recherche méthodologique pour la typologie de la végétation et la phytogéographie des forêts dense d'Afrique tropicale. Thèse de Doctorat Université libre de Bruxelles, Belgique, p. 372.

Siegel S, Castellan NJ.1988. Nonparametric Statistics for the Behavioral Sciences (2nd edn). McGraw-Hill Book Co: New York, USA.

Sonké B. 1996. Etudes floristiques et structurales des forêts dans la Réserve de Faune du Dja (Cameroun). Thèse de Doctorat, Université libre de Bruxelles, p. 255. 
Statistica. 2005. Statistica 6.0, version monoposte Window. Washington, D.C., US.

Tchouto MGP. 2004. Plant diversity in a Central African rain forest: implication for biodiversity conservation in Cameroon. Ph.D. thesis, Wageningen University, p. 208.

UNEP. 2004. World Conservation Monitoring Centre: protect areas programme. http://sea.unep-wcmc.org/sites/wh/dja. html

Van Gemerden BS, Olff H, Parren PE, Bongers F. 2003. The pristine rain forest? Remnants of historical human impacts on current tree species composition and diversity. J. Biogeography, 30: 13811390.

Vanleeuwe H, Gautier A. 1998. Forest elephant paths and movements at the Odzala National Park, Congo: The role of clearings and Marantaceae forest. Afr. J. Ecol., 36: 174-182.

Vansina J. 1991. Sur les sentiers du passé en forêt. Les cheminements de la tradition politique ancienne de l'Afrique équatoriale. Enquêtes et documents d'histoire Africaine, 9, AeaquatoriaUCL : Belgique.

Vermeulen C. 2000. Le facteur humain dans l'aménagement des espaces ressources en Afrique centrale forestière: application au Badjoué de l'Est -Cameroun. Thèse de Doctorat en Agronomie et Ingénierie Biologique, Faculté Universitaire des Sciences Agronomiques de Gembloux, p. 381 .
White F. 1983. The Vegetation of Africa. A descriptive memoir to accompany the UNESCO/AEFAT vegetation map of Africa. UNESCO: Paris France.

White L, Oslisly R. 1998. A window on the history of the central African rain forests. In La Gestion des Forêts Denses Africaines Aujourd'hui. Nasi R, Amsallem I, Drouineau S (eds). CDROM, FORAFRI: Libreville, Gabon.

White LJT, Edwards A. 2000. Conservation Research in the African Rain Forest: a Technical Handbook. The Wildlife Conservation Society: New York.

White LJT, Oates JF. 1999. New data on the history of the plateau forest of Okomu, southern Nigeria: insight into how human disturbance has shaped the African rain forest. Global Ecology and Biogeography, 8: 355-361.

White LJT. 1995. Factors affecting the duration of elephant dung-pile in rain forest in the Lope Reserve, Gabon. Afr. J. Ecol., 33: 142-150.

Williamson L, Usungo B. 1998. Résumé des inventaires biologiques: zoom sur le recensement de gorilles, chimpanzés, éléphants et singe diurnes du Dja. Canopée, 12: 4-5.

Willis KJ, Gilson L, Brncic, TM. 2004. How "Virgin" is virgin rainforest? Sciencemag, 304: 402-403.

WRI - GFW. 2005. Atlas forestier interactif du Cameroun. World Ressources Institute/ Global Forest Watch/MINEF. Waschington.D.C. et Yaoundé, Cameroun. www.wri.org; www. globalforestwatch.org 